\title{
Insulin protocol to control post-pancreatectomy diabetes
}

\author{
$\underline{\text { Shin Ae KANG* }}$ \\ Division of Endocrinology, Department of Internal Medicine, Gangnam Severance Hospital, \\ Yonsei University College of Medicine, Seoul, Korea
}

Lecture: Diabetes is a disease by which blood glucose level is elevated by defects in insulin secretion or by resistance to insulin. As pancreatectomy inevitably results in the reduction of the total islet mass, the majority of clinicians tended to believe that pancreatectomy itself would immediately cause blood glucose elevation and subsequent diabetes development. In partial pancreatectomy surgery (distal pancreatectomy or PPPD), however, not all patients develop diabetes for at least a number of years after. This is because the remnant pancreatic islets can produce relatively enough amount of insulin to maintain blood glucose level. After this compensatory time period passes after partial pancreatectomy, the pancreas eventually undergoes atrophy after surgery and the insulin secretory function decreases in the remnant islet. This will lead to delayed development of diabetes after pancreatectomy. Under these situations, insulin injection together with oral anti-diabetic medication would be critical for controlling the blood glucose level.

In contrast, patients who undergo total pancreatectomy will develop diabetes immediately after the surgery because of the absolute deficiency of insulin. In this situation, the patients will need a tightly-controlled, delicate insulin injection protocol with multiple subcutaneous insulin injections. Recently, with only a small patch applied to the body, continuous glucose monitoring system (CGMS) enables the reporting of the blood glucose level into the patient's smartphone every 1-5 minutes. The CGMS has gained popularity, is clinically helpful, and is even supported by the health insurance system for type 1 diabetes patients (the patients who undergoes total pancreatectomy can be categorized as type 1 diabetes by the Health Insurance Review System).

In this lecture, I will be addressing the different glucose management protocols and/or methods in a variety of pancreatectomy cases and will also introduce the usefulness of CGMS for total pancreatectomy patients. 